\title{
Clinical value of contrast-enhanced ultrasound in transthoracic biopsy of malignant anterior mediastinal masses
}

\author{
Jie Han ${ }^{1}$, Xiao-Li Feng ${ }^{2}$, Tian-Yu Xu ${ }^{1}$, Wen-Qi Feng ${ }^{1}$, Meng-Jia Liu ${ }^{1}$, Bo Wang ${ }^{1}$, Ting-Lin Qiu ${ }^{3}$, \\ Yong Wang ${ }^{1}$
}

${ }^{1}$ Department of Ultrasound, ${ }^{2}$ Department of Pathology, ${ }^{3}$ Cancer Quality Control Office, National Cancer Center/National Clinical Research Center for Cancer/Cancer Hospital, Chinese Academy of Medical Sciences and Peking Union Medical College, Beijing 100021, China

Contributions: (I) Conception and design: Y Wang; (II) Administrative support: Y Wang, TL Qiu; (III) Provision of study materials or patients: Y Wang; (IV) Collection and assembly of data: J Han, XL Feng; (V) Data analysis and interpretation: TL Qiu, J Han; (VI) Manuscript writing: All authors; (VII) Final approval of manuscript: All authors.

Correspondence to: Yong Wang. Department of Ultrasound, National Cancer Center/National Clinical Research Center for Cancer/Cancer Hospital, Chinese Academy of Medical Sciences and Peking Union Medical College, Beijing 100021, China. Email: drwangyong77@163.com; Ting-Lin Qiu. Cancer Quality Control Office, National Cancer Center/National Clinical Research Center for Cancer/Cancer Hospital, Chinese Academy of Medical Sciences and Peking Union Medical College, Beijing 100021, China. Email: qt1007@126.com.

Background: Given low incidence and high heterogeneity, the treatment strategies of anterior mediastinal masses (AMMs) are diverse based on pathology. The purpose of the study is to evaluate the usefulness of contrast-enhanced ultrasound (CEUS) in transthoracic biopsy of malignant AMMs when compared with that of ultrasound (US) alone and to screen lesions that are more suitable for CEUS evaluation and guidance.

Methods: We reviewed all the US- and CEUS-guided transthoracic core needle biopsy (CNB) of AMMs performed in National Cancer Center/National Clinical Research Center for Cancer/Cancer Hospital, Chinese Academy of Medical Sciences and Peking Union Medical College between July 2013 and April 2019. A total of 68 patients (mean age 36 years; male-female ration 1.6:1) who were suspected with malignant AMMs were enrolled in the study. Among them, 20 patients received pre-biopsy CEUS examination (CEUS group); 48 patients underwent conventional US examination and guidance (US group). Demographic, radiologic, pathologic, medical records, and biopsy procedure details were retrospectively reviewed and compared between the two groups.

Results: The display of internal necrosis areas was significantly improved when compared with that of the conventional US (70\%, 30\%; $\mathrm{P}=0.008)$. Specifically, CEUS improved the diagnostic accuracy of US-guided transthoracic biopsy $(95.0 \%, 79.2 \% ; \mathrm{P}=0.210)$ and especially for AMMs exceeding $10 \mathrm{~cm}(100 \%, 68.2 \%$; $\mathrm{P}=0.040)$ and carcinoma $(100 \%, 0 \% ; \mathrm{P}=0.048)$. The number of punctures in US group and CEUS group was 2.6 and 4.4 times, respectively $(\mathrm{P}<0.001)$. In case of similar number of punctures ( 1 to 3 times), CEUS improved diagnostic accuracy when compared to that of the conventional US $(100 \%, 75 \% ; \mathrm{P}=0.486)$. The technical success rate was $100 \%$ (68/68). In both groups, patients did not exhibit symptomatic complications such as bleeding, pneumothorax, or hemoptysis after the biopsy.

Conclusions: The application of CEUS in transthoracic biopsy of malignant AMMs improved diagnostic accuracy when compared with conventional US and especially played more important role in lesions exceeding $10 \mathrm{~cm}$ and presumptive clinical carcinoma.

Keywords: Contrast enhanced ultrasound (CEUS); ultrasound (US); anterior mediastinal masses (AMMs); biopsy

Submitted Sep 10, 2019. Accepted for publication Sep 20, 2019.

doi: $10.21037 /$ jtd.2019.11.51

View this article at: http://dx.doi.org/10.21037/jtd.2019.11.51

(c) Journal of Thoracic Disease. All rights reserved. 


\section{Introduction}

Anterior mediastinal masses (AMMs) are relatively uncommon, and extremely heterogeneous in origin and etiology and include epithelial, mesenchymal, lymphoid hematopoietic, and metastatic neoplasm. Some lesions that are typical benign as shown by imaging do not require treatment, while patients with clinical symptoms or suspected malignant AMMs should be treated actively. Treatment strategies are diverse and include surgery, chemotherapy, and/or radiotherapy based on specific pathologic diagnosis and clinical staging. Therefore, an accurate pre-treatment histopathologic diagnosis is crucial for patients.

There are several options to obtain tissue samples from mediastinal lesions for histopathologic diagnosis such as mediastinoscopy, mediastinotomy, open surgical biopsy, bronchoscopy, video-assisted thoracoscopic surgery (VATS), and image-guided transthoracic biopsy (1-3). Each technique exhibits its advantages and disadvantages in terms of accuracy, invasion, cost, and risk (4). Among the diagnostic techniques, image-guided transthoracic biopsy is less invasive and cost-effective when compared with other alternative methods. In current clinical practice, CT guidance still corresponds to the golden standard method and is widely acceptable for chest physicians (5). Given the advantages of nonradiative exposure, mobile machinery, real-time mode, and cost-effectiveness, US guidance is more preferable if the "target" lesion is adjacent to the thoracic wall or thoracic inlet and is adequately displayed by US (6). Based on previous studies, US guidance of mediastinal and peripheral intrathoracic biopsy was similar or even higher in diagnostic samples yield when compared with CT guidance with less complications (1,7-9). The diagnostic failure of US-guided biopsy of AMMs is mainly due to tumor intrinsic heterogeneity and results in insufficient sampling and inconclusive diagnosis. Fortunately, contrast-enhanced ultrasound (CEUS) aids clinicians in differentiating viable and nonviable zones (necrosis or fibrosis) with considerable confidence to improve diagnostic efficacy. Additionally, CEUS is introduced in the field of interventional ultrasound (US), and a biopsy from viable areas can increase diagnostic yield by $10 \%$ and decrease the false negative rate and especially in larger tumors with necrotic areas (10).

The purpose of the retrospective study involved comparing the value of CEUS $v s$. US in transthoracic biopsy of AMMs and to screen the lesions that are more suitable for CEUS evaluation and guidance.

\section{Methods}

\section{Patients}

From July 2013 to April 2019, 68 patients who were suspected with malignant AMMs by CT/MRI/PETCT and underwent US- or CEUS-guided transthoracic core needle biopsy $(\mathrm{CNB})$ were enrolled in the study. The patients included 42 males and 26 females (1.6:1) with a mean age of 36 (range, 14-68) years.

The indications for US guidance were as follows: (I) the biopsy target was adjacent to the thoracic wall; (II) the safe puncture site and path confirmed by US intercostal scanning. Exclusion criteria: incomplete medical records and $<5 \mathrm{~cm}$ AMMs. Additionally, CEUS-guided transthoracic biopsy was performed in our department since September 2015. In our earlier experience, high diagnostic accuracy is achieved in $<5 \mathrm{~cm}$ AMMs due to less necrosis, and thus CEUS guidance was not recommended in smaller lesions in clinical practice.

Seven patients were observed with incident discovery of AMMs without symptoms on routine physical examination, and the others exhibited symptoms such as cough (45.6\%), chest distress and pain (42.6\%), fever (17.6\%), dyspnea $(10.3 \%)$, swelling of the head and face $(5.9 \%)$, hoarseness of voice $(2.9 \%)$, dysphagia (2.9\%), and hemoptysis $(1.5 \%)$. Two patients (one thymic squamous cell carcinoma and one neuroendocrine tumor) received re-biopsy for a follow-up therapeutic schedule after chemoradiotherapy.

All patients did not exhibit absolute contraindications to biopsy (bleeding tendency) or contraindications to US contrast agent SonoVue ${ }^{\circledR}$ (Bracco, Milan, Italy) (e.g., severe cardiopulmonary diseases or allergic reactions).

All patients or legal guardians signed informed consent prior to CEUS examination and biopsy procedure. The study was approved by the Institutional Ethics Review Board (National Cancer Center/National Clinical Research Center for Cancer/Cancer Hospital, Chinese Academy of Medical Sciences and Peking Union Medical College NCC2016YZ-15).

\section{Pre-biopsy US examination}

The patients were placed in a supine position or a semiupright position due to dyspnea in the horizontal position.

All examinations were performed using Philips iU22 (Philips, USA), Philips EPIQ5 (Philips, USA), or Aloka Prosounda10 (Aloka, Japan) with a convex array probe C5-2, 
Table 1 Baseline characteristics of patients in the study

\begin{tabular}{lccc}
\hline Characteristics & US group $(n=48)$ & CEUS group $(n=20)$ & $P$ value \\
\hline Age (year) & $37 \pm 15[14-68]$ & $35 \pm 13[18-63]$ & 0.615 \\
Male/female, $n$ & $27 / 21$ & $15 / 5$ & 0.147 \\
Lesion size $(\mathrm{cm})$ & $9.8 \pm 3.0[5.3-17.5]$ & $11.1 \pm 3.2[6.4-17.6]$ & 0.120 \\
\hline
\end{tabular}

US, ultrasound; CEUS, contrast enhanced ultrasound.

C5-1, or C5-3. Grayscale and color Doppler US were routinely performed to evaluate the size, internal echo, and vascularity of the lesion.

In the US group, we selected the solid part of the lesion or the rim region of the large tumors as the optimal puncture target and avoided large blood vessels along the puncture pathway. In the CEUS group, a bolus of 2.4-mL SonoVue ${ }^{\circledR}$ (Bracco, Milan, Italy) was injected into the antecubital vein, and this was followed by a $5-\mathrm{mL}$ flush of saline. The perfusion of the lesion was scanned continuously for at least $3 \mathrm{~min}$. The dynamic image was recorded on the hard disk of the US system. The enhancement region without necrosis was determined as the puncture target.

\section{CNB procedure}

Most biopsy procedures were performed with a freehand technique. An automatic biopsy gun (Bard Biopsy System, Tempe, AZ, USA) was used while 16- or 18-gage biopsy needle selection was based on the preference of the performing doctors.

The common puncture site corresponded to right or left parasternal or intercostal approaches based on the location and the extension of the lesion. The skin was sterilized and local anesthesia was applied at the puncture site and along the pathway using $1 \%$ lidocaine before the biopsy procedures. Subsequently, a core biopsy needle coupled on a BARD automatic biopsy gun was inserted in the previously determined puncture target. The complete procedure was monitored in real time to avoid vessels and adjust the pathway. In the CEUS group, the lesions exhibiting heterogenous enhancement with large necrotic area were given a second $1.2-\mathrm{mL}$ dose of SonoVue ${ }^{\circledR}$ for real-time CEUS guidance. The number of punctures was determined by the quantity and color of the sample obtained. The sampling tissues were fixed in $10 \%$ formalin and sent for histology and immune-histochemistry diagnosis as performed by two experienced pathologists.

The patients were observed for $1-2 \mathrm{~h}$ after the procedure. Patients did not exhibit significant complications such symptomatic bleeding, pneumothorax, or hemoptysis.

\section{Final diagnosis}

The final diagnosis was determined based on the following criteria: (I) if the patient underwent the surgical treatment, then surgical pathology corresponded to the final diagnosis; (II) if the biopsy result revealed a specific malignant pathology such as thymoma or Hodgkin lymphoma, then it was accepted as the final diagnosis; (III) in case of nonspecific pathology (e.g., carcinoma, malignant, benign, etc.) or suspicious pathology with additional information (e.g., inadequate tissue, tissue degeneration, etc.), the clinical comprehensive diagnosis was considered as the final diagnosis. The diagnostic accuracy of biopsy was defined as the percentage of the lesions that received specific pathology diagnosis.

\section{Statistics}

Statistical analysis was performed via SPSS version 19 software (IBM Corp., Armonk, NY, USA). Categorial data was expressed as percentages; Continuous data with normal distribution was expressed as mean \pm standard deviation, otherwise as median. The difference in lesion size between the two groups was analyzed via the Independent-Samples $T$ test. The $\chi^{2}$-test, Fisher's exact test, and McNemar test were used to analyze differences in categorical variables between groups. Additionally, $\mathrm{P}<0.05$ was considered as statistically significant.

\section{Results}

Twenty patients who received CEUS before or throughout whole biopsy procedure were defined as CEUS group. Forty-eight patients who only underwent conventional US were defined as US group. There was no statistically significant difference in patients' age, male to female ratio, or lesion size between US and CEUS group (Table 1).

In the CEUS group, there were 14 (70\%) heterogenous solid masses, 4 (20\%) cystic and solid masses, and two (10\%) predominantly solid masses with little necrotic area. After the contrast enhanced agent injection, 6 lesions exhibited homogeneous enhancement without non-enhanced area (Figure 1). Among the other 14 lesions, eight lesions showed non-liquefied necrotic areas that appeared as hypoechoic or isoechoic on B-mode US, four exhibited a larger non- 

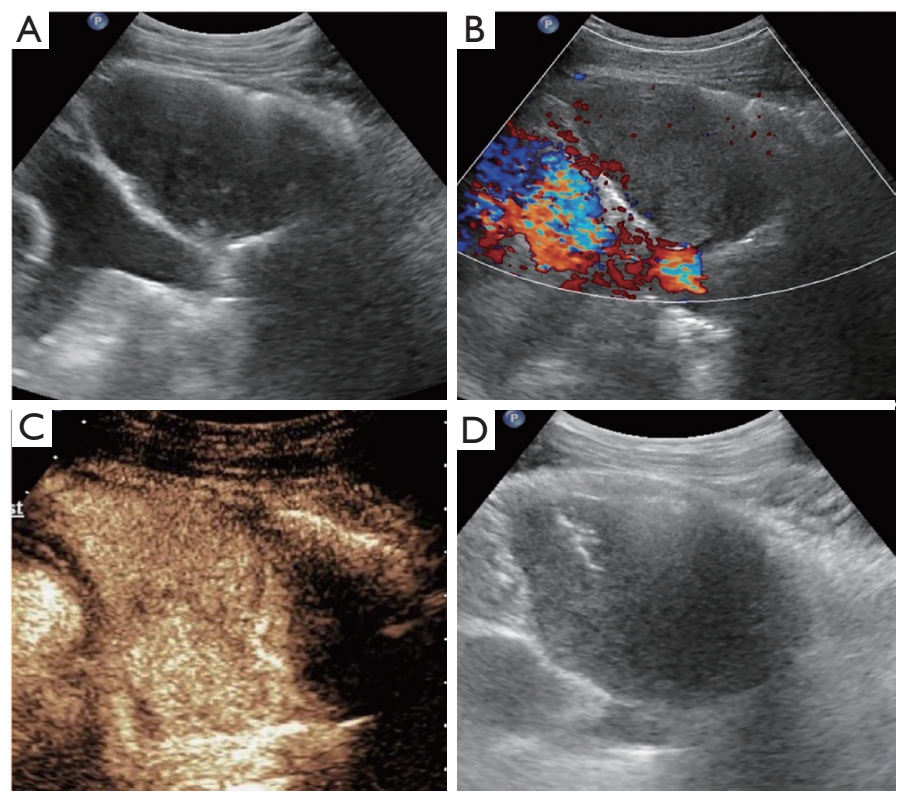

Figure 1 Metastasis of rectal cancer in a 63-year-old man. (A) Grayscale ultrasound image shows a well-defined homogeneous hypoechoic mass; (B) color Doppler flow image shows the absence of vessels in the mass; (C) CEUS shows homogenous hyperenhancement; (D) biopsy is directly performed with an 18-gage automatic needle by B-mode ultrasound after CEUS evaluation. CEUS, contrast enhanced ultrasound.

enhanced necrotic area when compared to that detected as anechoic area via B-mode US (Figure 2), and necrotic area was similar in two lesions. The display of internal necrosis areas was significantly improved when compared to conventional US $(70 \%, 30 \% ; \mathrm{P}=0.008)$. However, real-time CEUS-guided biopsy was performed in eight cases, and 12 patients underwent real-time B-mode guidance without a second SonoVue ${ }^{\circledR}$ injection for the overlap of the puncture pathway under US and CEUS evaluation (Figure 3).

Based on the final diagnosis, lymphoma $(45.6 \%)$ was the most common entity, and this was followed by thymic carcinoma $(22.1 \%)$ and germ cell tumor $(14.7 \%)$. The age onset of lymphoma and germ cell tumor was younger than that of thymic carcinoma. Table 2 lists the age distribution of the patients along with different AMMs.

In the US group, 79.2\% (38/48) cases achieved specific diagnosis with sufficient information for therapy. The other $10(20.8 \%)$ cases were accepted biopsy diagnostic failure based on the final diagnosis (Table 3). The diagnostic accuracy of CEUS group was $95.0 \%$ (19/20). However, the difference in diagnostic accuracy between the two groups was not statistically significant $(\mathrm{P}=0.210)$. The cases were further divided based on lesion size. There was significant difference in diagnostic accuracy between US and CEUS group for lesions exceeding $10 \mathrm{~cm}(\mathrm{P}=0.040)$ while no statistically significant difference was observed in $<10 \mathrm{~cm}$ lesions $(\mathrm{P}=0.732)$ (Table 4). With respect to the histologic diagnosis, the percentage of definite diagnosis for carcinoma group in CEUS group exceeded that of US group $(\mathrm{P}=0.048)$ although no statistically significant difference was observed for non-carcinoma group $(\mathrm{P}=0.430)$ (Table 5).

An 18-gage biopsy needle was mostly used (86.8\%), and only nine cases $(13.2 \%)$ chose 16-gage biopsy core needle. The number of punctures in US group and CEUS group was 2.6 and 4.4 times, respectively $(\mathrm{P}<0.001)$. In the US group, punctures were performed 1 to 3 times in 10 failure cases while all cases with a specific diagnosis exhibited a similar number of punctures in CEUS group $(75 \%, 100 \%$; $\mathrm{P}=0.486)$. The technical success rate was $100 \%(68 / 68)$. In both groups, patients did not exhibit symptomatic complications such as bleeding, pneumothorax, or hemoptysis after the biopsy.

\section{Discussion}

With the increasing use of chest computed tomography (CT) in health checkup and clinical practice, asymptomatic incidental AMMs are becoming common. And a considerable portion of the AMMs are suspected to be benign such as thymic cyst or hyperplasia (absence 

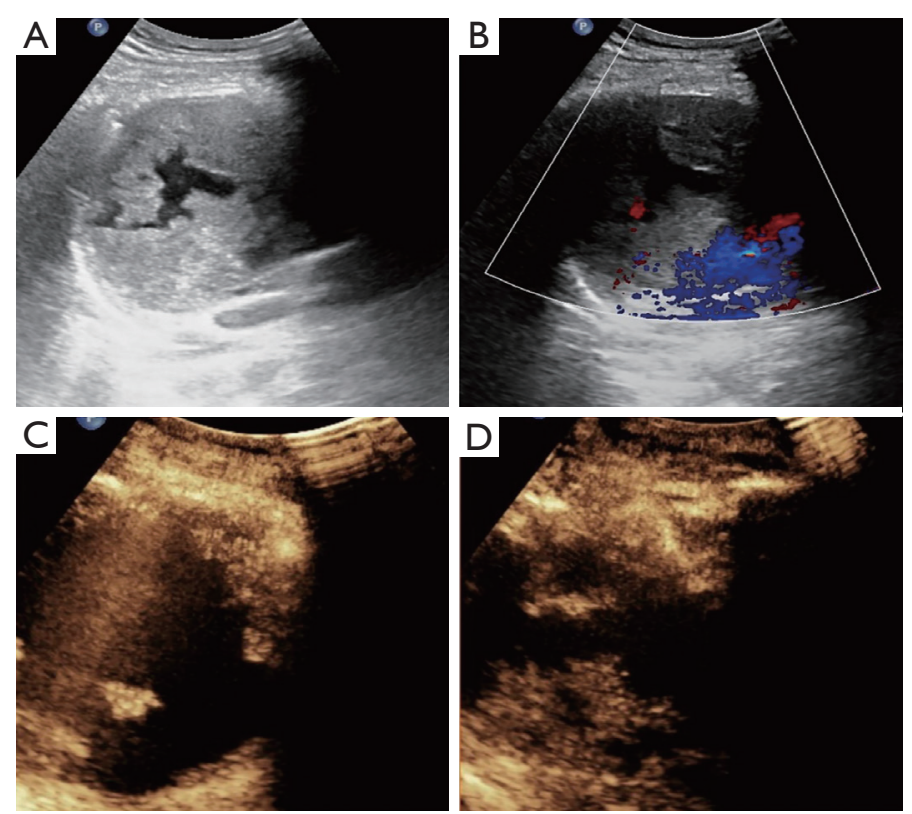

Figure 2 Right anterior mediastinal mass in a 33-year-old man. Biopsy is performed with an 18-gage automatic needle guided by CEUS. Pathologic diagnosis corresponds to malignant mixed germ cell tumor, mainly yolk sac tumor. (A) Grayscale ultrasound image shows a welldefined heterogeneous hypoechoic mass with necrosis; (B) color Doppler flow image shows the absence of vessels in the mass; (C) CEUS shows larger non-enhanced necrotic area after contrast agent injection; (D) CEUS-guided core needle biopsy is focused on the hyperenhanced area and avoids the anechoic region. CEUS, contrast enhanced ultrasound.
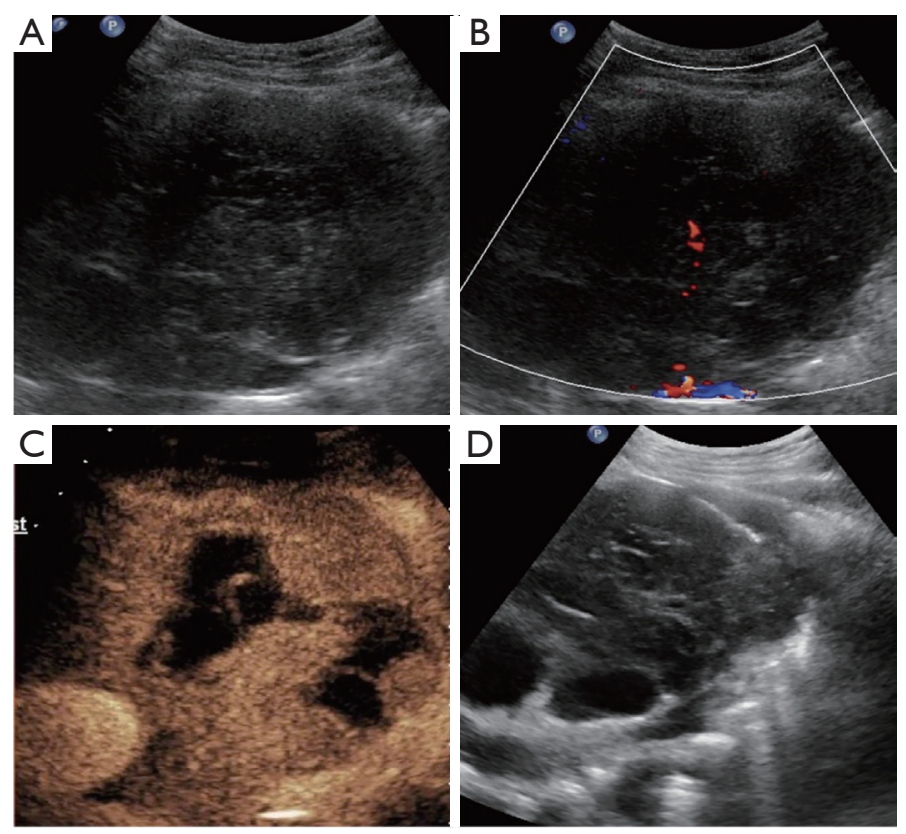

Figure 3 Diffuse large B cell lymphoma in a 37-year-old woman. (A) Grayscale ultrasound image shows an ill-defined heterogeneous hypoechoic mass; (B) color Doppler flow image shows few vessels in the mass; (C) CEUS shows non-liquefied necrotic areas; (D) biopsy is performed with an 18-gage automatic needle guided by B-mode ultrasound focusing on the rim region of the lesion which showed hyperenhancement in CEUS. CEUS, contrast enhanced ultrasound. 
Table 2 Distribution of anterior mediastinal masses (AMMs) in relation to age group

\begin{tabular}{|c|c|c|c|c|c|c|c|}
\hline Final diagnosis & \multicolumn{6}{|c|}{ Age range (years) } & Total \\
\hline Lymphoma & 6 & 10 & 8 & 6 & 1 & & 31 \\
\hline Non-Hodgkin's lymphoma & 4 & 10 & 6 & 3 & 1 & & 24 \\
\hline Hodgkin's lymphoma & 2 & & 2 & 3 & & & 7 \\
\hline Thymic tumor & 1 & 1 & 1 & 1 & 1 & & 5 \\
\hline Thymic carcinoma & & 2 & & 4 & 7 & 2 & 15 \\
\hline Lung cancer & & & 1 & & 1 & & 2 \\
\hline Neuroendocrine carcinoma & & & & 1 & 1 & & 2 \\
\hline Total & 10 & 18 & 12 & 13 & 11 & 4 & 68 \\
\hline
\end{tabular}

Table 3 Biopsy diagnostic failure of anterior mediastinal masses (AMMs) (11 cases)

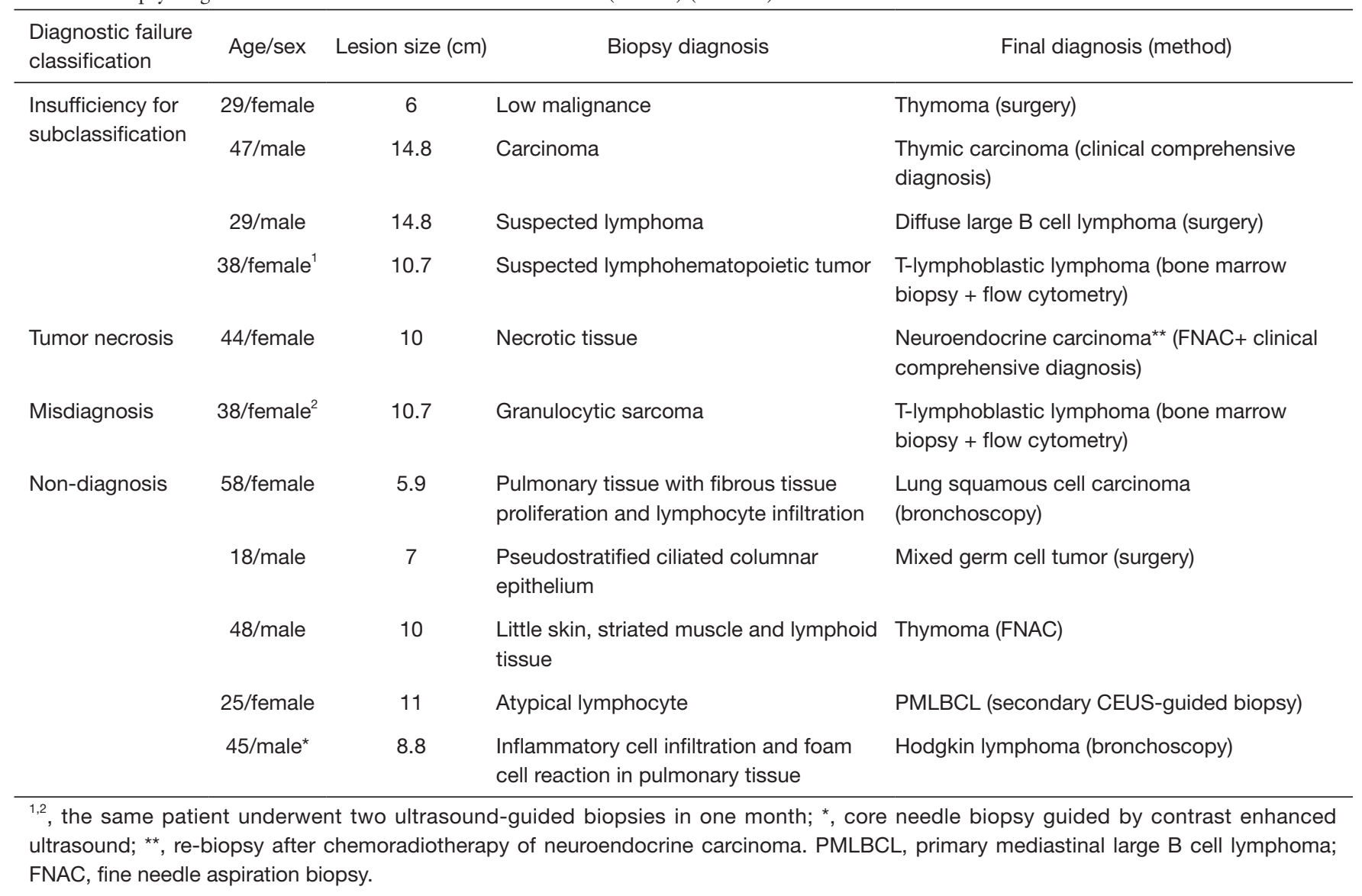


of myasthenia gravis), which just need to manage conservatively with follow-up (11). AMMs include a variety of diseases, such as primary and secondary, infectious and neoplastic, benign and malignant. Early diagnosis and treatment are crucial in the management of patients with malignant AMMs. In addition to the directly resectable lesions, a minimally invasive technique that provides a definitive diagnosis is typically preferred. An accurate histological diagnosis is essential for a proper regimen and even for the preoperative neoadjuvant therapy (12). Inconclusive diagnosis delays specific therapy and also increases the necessity of more invasive diagnostic procedures and prolongs hospital stays (13).

US is considered as a "Gold Standard" for procedure guidance if the target lesion can be adequately imaged (1). Based on the previous reports, the diagnostic accuracy of US-guided transthoracic biopsy varies from $72.1 \%$ to $98 \%$ $(2,7,9,14,15)$. Additionally, the common reason for CNB diagnostic failure corresponds to tumor necrosis, inadequate sampling, or sampling error (4). Conventional US does not show the non-liquefied necrosis. However, tissue necrosis

Table 4 Diagnostic accuracy between the US group and CEUS group

\begin{tabular}{lccc}
\hline \multirow{2}{*}{ Groups } & \multicolumn{2}{c}{ Diagnostic accuracy \% } & \multirow{2}{*}{ P value } \\
\cline { 2 - 3 } & US group & CEUS group & \\
\hline$<10 \mathrm{~cm}$ & $88.5(23 / 26)$ & $88.9(8 / 9)$ & 0.732 \\
$\geq 10 \mathrm{~cm}$ & $68.2(15 / 22)$ & $100(11 / 11)$ & 0.040 \\
Total & $79.2(38 / 48)$ & $95.0(19 / 20)$ & 0.210 \\
\hline
\end{tabular}

US, ultrasound; CEUS, contrast enhanced ultrasound. and viability can be evaluated effectively with CEUS, and biopsy from perfused areas results in a $10 \%$ to $15 \%$ increase in diagnostic accuracy (16). In the present study, the display of internal necrosis areas is significantly improved by $40 \%$ after contrast agent injection; and the CEUS group exhibited a $15.8 \%$ increase in diagnostic accuracy. However, the difference was not statistically significant. We believe this is due to the relatively small number of patients in CEUS group, and a prospective trial randomizing involving larger samples can be helpful in confirming the value of CEUS in transthoracic biopsy of AMMs.

Given the unbalance between the growth of supplying vessels and the fast growth of tumor, tissue liquefaction and necrosis is more likely to be observed in larger and malignant lesions $(17,18)$. Based on previous studies and our early experience, a CEUS examination prior to the biopsy procedure was recommended to patients with bigger lesion $(>5 \mathrm{~cm})$. Despite the lack of statistical difference in $5-10 \mathrm{~cm}$ lesions, our study results confirmed the value of CEUS in improving diagnostic accuracy in lesions exceeding $10 \mathrm{~cm}$. The result exhibited the same tendency as the results in previous studies, thereby CEUS improved the diagnostic accuracy when the lesion size increased when compared to conventional US $(15,17)$. Nevertheless, it was not fully consistent with the results obtained by Fu et al. (15) and Wang et al. (17), and the most likely reason corresponded to the increase in bulky masses included in the present study. Furthermore, we divided AMMs into carcinoma and non-carcinoma groups, and there were only two cases of carcinoma in US group and five in CEUS group. Thus, CEUS is helpful in improving the diagnostic accuracy and especially in carcinoma group $(\mathrm{P}=0.048)$. However, it is

Table 5 Diagnostic accuracy of noncancerous and cancerous lesions in the US group and CEUS group

\begin{tabular}{lccc}
\hline \multirow{2}{*}{ Final diagnosis } & \multicolumn{3}{c}{ Diagnostic accuracy of biopsy, $\mathrm{n}(\%)$} \\
\cline { 2 - 3 } & US group & CEUS group & P value \\
\hline Non-carcinoma & $38 / 46(82.6 \%)$ & $14 / 15(93.3 \%)$ & 0.430 \\
Lymphoma & $17 / 21(81.0 \%)$ & $9 / 10(90.0 \%)$ & 0.472 \\
Thymic tumor & $14 / 17(82.4 \%)$ & $3 / 3(100 \%)$ & 0.596 \\
Germ cell tumor & $7 / 8(87.5 \%)$ & $2 / 2(100 \%)$ & 0.800 \\
Carcinoma* & $0 / 2(0 \%)$ & $5 / 5(100 \%)$ & 0.048 \\
Total & $38 / 48(79.2 \%)$ & $19 / 20(95.0 \%)$ & 0.210 \\
\hline
\end{tabular}

*, included 1 lung adenocarcinoma; 1 lung squamous cell carcinoma; 2 neuroendocrine carcinomas; 1 esophageal squamous cell carcinoma; 1 metastasis of rectal carcinoma; 1 metastasis of lung squamous cell carcinoma. US, ultrasound; CEUS, contrast enhanced ultrasound. 
possible for a higher patient population with different type of AMMs pathology to exhibit an increased tendency and aid specialists in implementing special workups for different patients.

In our study, all lesions were malignant. Based on the final diagnosis, lymphoma was the most common malignancy and accounted for $45.6 \%$ of the patients, and this was followed by thymic carcinoma and germ cell tumor. The only undetected in CEUS group corresponded to a 45 year old male with mixed cellular Hodgkin's lymphoma. Isolated Reed-Sternberg cells in an appropriate background that are sparse or unevenly distributed are required to diagnose Hodgkin's lymphoma. However, patchy or scanty $\mathrm{R}-\mathrm{S}$ cells result in higher inconclusive diagnosis (19-21). In this case, three cores in the fast washout area and four cores in the late washout area resulted in negative. Abundant inflammatory cells and foam cells, rare R-S cells, affected lung tissue and were considered as the most likely reason for false negative.

In our cohort, two patients received two biopsies although each biopsy procedure was treated independently in terms of statistics. Their final diagnosis was lymphoma. One of the patients was a $25-\mathrm{y}$ old female. Both biopsies chose an 18-gage core needle. The first attempt involved a conventional US without diagnosis due to inadequate tissue with large necrosis. The second attempt involved CEUS guidance twenty days later, and the specific of primary mediastinal large B cell lymphoma was obtained. The other patient was a 38-y old female, and both attempts were under conventional US guidance. The first biopsy punctured one time while using an 18-gage core needle, and the immunohistology involved suspected lymphohematopoietic tumor albeit lack of confidence due to an insufficient tissue sample. The second biopsy was received thirteen days later with a $16 \mathrm{G}$ core needle thrice although the immunohistology indicated misdiagnosis of granulocytic sarcoma. Subsequently, the final diagnosis of T-lymphoblastic lymphoma was received via bone marrow biopsy and flow cytometry technique. The experience of the two patients indicates that the application of CEUS in re-biopsy is potentially a better choice when compared to the use of larger core needle for those with an unsuccessful diagnosis at the first puncture. Additionally, several previous studies suggest that larger needle size plays a slightly role for CNB of lymphoma (21-24).

In terms of number of punctures, there were more attempts in the CEUS group when compared with US group, and this was inconsistent with the results of previous studies $(2,14,15,17,18)$. There are several possible reasons for this result. Based on the medical records, five patients (25\%) in the CEUS group accepted biopsy at least once prior to the CEUS evaluation albeit without successful diagnosis. Thus, the patients' lesions in CEUS group were more difficult in terms of pathologic diagnosis. Previous reports indicate that multiple cores from different areas of lesion are crucial for a high yield diagnosis (21). Given the consideration of obtaining adequate tissue samples for accurate diagnosis and avoiding more repeated biopsy punctures, the operator performed two to seven attempts per each lesion in CEUS group. However, in the case of similar number of punctures, CEUS improved $25 \%$ diagnostic accuracy when compared to that of the conventional US.

There are several limitations in the study. First, the study was retrospective. The proportion of CEUS group was low, and the application of CEUS was not random, and thus it was based on the recommendation of the specialist and patient's agreement. Real-time CEUS guidance was not performed in all CEUS group cases. Given the tumor specialized hospital, a few patients who attended to our institution already accepted biopsy at local hospital albeit without successful pathologic diagnosis. Thus, this need not correspond to the first biopsy for patients. Finally, all biopsies in CEUS group were performed by the same doctor while the US-guided biopsies were randomly distributed.

In an era of personalized and precision medicine, we recommend CEUS evaluation or guidance that need not be routinely performed in transthoracic biopsy for AMMs based on additional intravenous contrast agent injection and cost-effectiveness principle and instead should be based on the tumor characteristics, and especially for lesions exceeding $10 \mathrm{~cm}$ and presumptive clinical carcinoma. A real-time CEUS guidance is not necessary when the biopsy route overlaps with conventional US at the preliminary CEUS evaluation.

\section{Acknowledgments}

Funding: The study was supported by Beijing Hope Run Special Fund of Cancer Foundation of China (LC2016A04).

\section{Footnote}

Conflicts of Interest: The authors have no conflicts of interest to declare. 
Ethical Statement: The authors are accountable for all aspects of the work in ensuring that questions related to the accuracy or integrity of any part of the work are appropriately investigated and resolved. The study was approved by the Institutional Ethics Review Board (National Cancer Center/National Clinical Research Center for Cancer/Cancer Hospital, Chinese Academy of Medical Sciences and Peking Union Medical College NCC2016YZ-15).

\section{References}

1. Azrumelashvili T, Mizandari M, Magalashvili D, et al. Imaging guided mediastinal percutaneal core biopsy-technique and complications. Georgian Med News 2015:24-34.

2. Zhou JH, Shan HB, Ou W, et al. Contrast-Enhanced Ultrasound Improves the Pathological Outcomes of USGuided Core Needle Biopsy That Targets the Viable Area of Anterior Mediastinal Masses. Biomed Res Int 2018;2018:9825709.

3. Carter BW, Marom EM, Detterbeck FC. Approaching the patient with an anterior mediastinal mass: a guide for clinicians. J Thorac Oncol 2014;9:S102-9.

4. Chockalingam A, Hong K. Transthoracic needle aspiration: the past, present and future. J Thorac Dis 2015;7:S292-9.

5. Görgülü FF, Oksuzler FY, Arslan SA, et al. Computed tomography-guided transthoracic biopsy: Factors influencing diagnostic and complication rates. J Int Med Res 2017;45:808-15.

6. Stigt JA, Groen HJ. Percutaneous ultrasonography as imaging modality and sampling guide for pulmonologists. Respiration 2014;87:441-51.

7. Jarmakani M, Duguay S, Rust K, et al. Ultrasound Versus Computed Tomographic Guidance for Percutaneous Biopsy of Chest Lesions. J Ultrasound Med 2016;35:1865-72.

8. Koegelenberg CF, Diacon AH, Irusen EM, et al. The diagnostic yield and safety of ultrasound-assisted transthoracic biopsy of mediastinal masses. Respiration 2011;81:134-41.

9. Rubens DJ, Strang JG, Fultz PJ, et al. Sonographic guidance of mediastinal biopsy: an effective alternative to CT guidance. AJR Am J Roentgenol 1997;169:1605-10.

10. Cui NY, Liu JY, Wang Y, et al. Contrast enhanced ultrasound guided biopsy shows higher positive sampling rate than conventional ultrasound guided biopsy for gastrointestinal stromal tumors diagnosis. Transl Cancer Res 2016;5:152-9.

11. Sun Q, Ku X, Xu N, et al. Investigation of an optimal lysis method for the study of thymus and thymoma by mass spectrometry-based proteomics. Transl Cancer Res 2018;7:391-400.

12. Lim W, Ridge CA, Nicholson AG, et al. The 8(th) lung cancer TNM classification and clinical staging system: review of the changes and clinical implications. Quant Imaging Med Surg 2018;8:709-18.

13. Nasit JG, Patel M, Parikh B, et al. Anterior mediastinal masses: A study of 50 cases by fine needle aspiration cytology and core needle biopsy as a diagnostic procedure. South Asian J Cancer 2013;2:7-13.

14. Yi D, Feng M, Wen Ping W, et al. Contrast-enhanced USguided percutaneous biopsy of anterior mediastinal lesions. Diagn Interv Radiol 2017;23:43-8.

15. Fu J, Yang W, Wang S, et al. Clinical value of contrastenhanced ultrasound in improving diagnostic accuracy rate of transthoracic biopsy of anterior-medial mediastinal lesions. Chin J Cancer Res 2016;28:617-25.

16. Nolsøe CP, Nolsoe AB, Klubien J. Use of Ultrasound Contrast Agents in Relation to Percutaneous Interventional Procedures: A Systematic Review and Pictorial Essay. J Ultrasound Med 2018;37:1305-24.

17. Wang S, Yang W, Zhang H, et al. The Role of ContrastEnhanced Ultrasound in Selection Indication and Improveing Diagnosis for Transthoracic Biopsy in Peripheral Pulmonary and Mediastinal Lesions. Biomed Res Int 2015;2015:231782.

18. Cao BS, Wu JH, Li XL, et al. Sonographically guided transthoracic biopsy of peripheral lung and mediastinal lesions: role of contrast-enhanced sonography. J Ultrasound Med 2011;30:1479-90.

19. Skelton E, Jewison A, Okpaluba C, et al. Imageguided core needle biopsy in the diagnosis of malignant lymphoma. Eur J Surg Oncol 2015;41:852-8.

20. Hu Q, Naushad H, Xie Q, et al. Needle-core biopsy in the pathologic diagnosis of malignant lymphoma showing high reproducibility among pathologists. Am J Clin Pathol 2013;140:238-47.

21. Groneck L, Quaas A, Hallek M, et al. Ultrasound-guided core needle biopsies for workup of lymphadenopathy and lymphoma. Eur J Haematol 2016;97:379-86.

22. Huang PC, Liu CY, Chuang WY, et al. Ultrasoundguided core needle biopsy of cervical lymphadenopathy in patients with lymphoma: the clinical efficacy and factors 
associated with unsuccessful diagnosis. Ultrasound Med Biol 2010;36:1431-6.

23. Burke C, Thomas R, Inglis C, et al. Ultrasound-guided core biopsy in the diagnosis of lymphoma of the head and neck. A 9 year experience. Br J Radiol 2011;84:727-32.

Cite this article as: Han J, Feng XL, Xu TY, Feng WQ, Liu MJ, Wang B, Qiu TL, Wang Y. Clinical value of contrastenhanced ultrasound in transthoracic biopsy of malignant anterior mediastinal masses. J Thorac Dis 2019;11(12):5290-5299. doi: $10.21037 /$ jtd.2019.11.51
24. Colella S, Scarlata S, Bonifazi M, et al. Biopsy needles for mediastinal lymph node sampling by endosonography: current knowledge and future perspectives. J Thorac Dis 2018;10:6960-8. 